\title{
Wide alternating QRS complexes from herbal poisoning
}

\author{
Saeed AIQahtani, MBBS*+‡; Jonathan Zipursky, MD*†; Omid Salehian, MSc, MD; \\ Margaret Thompson, $\mathrm{MD}^{*}+$
}

Keywords: Herbal poisoning, aconite, poisoning, toxicity, ventricular dysrhythmia

\section{CASE REPORT}

A 44-year-old male with a history of hypertension and chronic kidney disease presented to the emergency department (ED) after an out-of-hospital cardiac arrest. The patient had an unknown downtime prior to paramedic arrival and initiation of cardiopulmonary resuscitation. The first rhythm noted on the automatic external defibrillator was pulseless electrical activity. Return of spontaneous circulation was achieved, and the patient was transferred to the ED where initial laboratory tests revealed K $6.1 \mathrm{mmol} / \mathrm{L}$, pH 7.2, HCO3 $16 \mathrm{mmol} / \mathrm{L}$, creatinine $660 \mathrm{mmol} / \mathrm{L}$ (baseline creatinine $500 \mathrm{mmol} / \mathrm{L}$ ). Initial hyperkalemia management included calcium gluconate, sodium bicarbonate, and insulin with dextrose. Hemodialysis was started urgently because of the metabolic acidosis and hyperkalemia in the setting of persistent dysrhythmias. Despite improvement in metabolic abnormalities following hemodialysis, the patient continued to have ongoing ventricular tachydysrhythmias, which were refractory to electrical cardioversion and intravenous amiodarone. An electrocardiogram (ECG) was obtained (Figure 1).

\section{QUESTION}

What is the ECG diagnosis, and what could cause this condition?

\section{INTERPRETATION AND ECG DIAGNOSIS}

The ECG demonstrates wide QRS complexes and left bundle branch block with alternating left and extreme right axes, consistent with a diagnosis of bidirectional ventricular tachycardia. Bidirectional ventricular tachycardia is defined as wide QRS rhythm with any of the following: 1) alternating left and right bundle branch block, 2) right bundle branch block with alternating left-and-right axis deviation, or 3) beat-to-beat variation of frontal QRS axis.

\section{CLINICAL COURSE}

The ECG findings of bidirectional ventricular tachycardia prompted empiric treatment with DigiFab ${ }^{\circledR}$ (digoxin immune FAB antidote) in consultation with the local poison centre. However, there was no clinical response, and, once available, the pretreatment serum digoxin level was undetectable. Further history from the family revealed that earlier in the day, the patient had ingested a large amount of raw aconite root. He had previously steeped the aconite root in a tea and would drink it daily to relieve arthralgias. The confirmative testing for aconite exposure was not available, and supportive care was continued for the presumed diagnosis of aconite poisoning. After 24 hours, the ventricular dysrhythmias resolved with eventual improvement in the hemodynamic status and decreased requirements of inotropes and vasopressors. However, despite ongoing supportive care, the neurologic exam and neuroimaging findings were consistent with brain death secondary to anoxic

From the *Division of Clinical Pharmacology and Toxicology, University of Toronto, Toronto, ON; †Ontario and Manitoba Poison Centres, Toronto, ON; ¥Department of Emergency Medicine, King Abdulaziz Medical City, Riyadh, Saudi Arabia; and the §Division of Cardiology, Department of Medicine, McMaster University, Hamilton, ON.

Correspondence to: Dr. Saeed AIQahtani, Division of Clinical Pharmacology and Toxicology, University of Toronto, Ontario Poison Centre, 555 University Avenue, Toronto, ON M5 G 1X8; Email: saeed.alqahtani@medportal.ca

(C) Canadian Association of Emergency Physicians

CJEM 2019;21(5):676-679

DOI 10.1017/cem.2019.366 


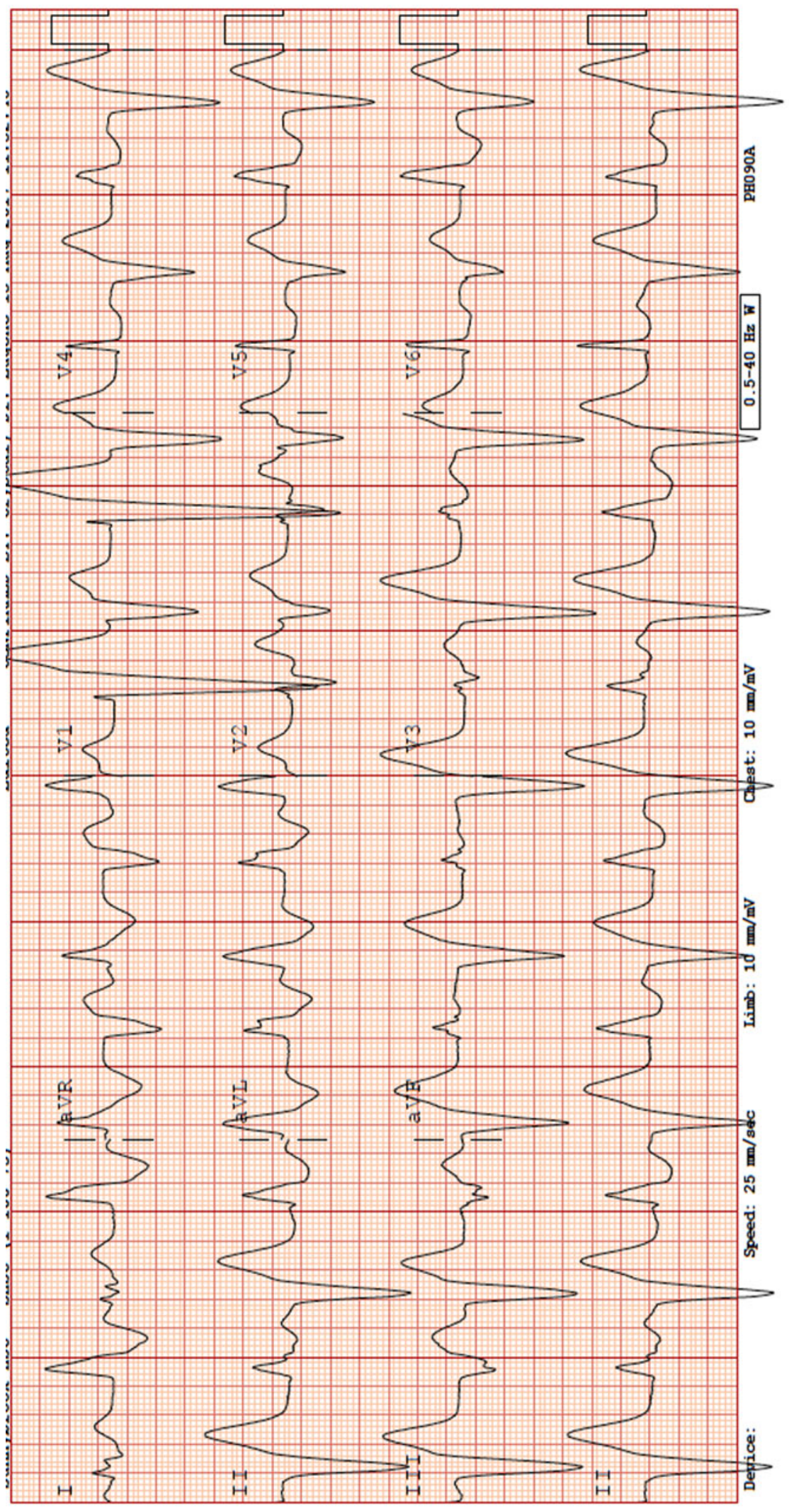


brain injury. The family subsequently decided to withdraw care.

\section{DISCUSSION}

Bidirectional ventricular tachycardia is a rare ventricular dysrhythmia characterized by beat-to-beat variation in the QRS axis. The differential diagnosis for this dysrhythmia is limited to severe digitalis/digoxin toxicity, ${ }^{1}$ familial catecholaminergic polymorphic ventricular tachycardia, ${ }^{2}$ and herbal aconite poisoning. ${ }^{3}$ Importantly, if bidirectional ventricular tachycardia is recognized on ECG, digoxin toxicity should always be at the top of the differential diagnosis because it is this poisoning alone that has antidotal treatment. Consideration should be given to catecholaminergic polymorphic ventricular tachycardia and aconite toxicity as possible etiologies once digoxin toxicity has been ruled out.

Aconite (aconitum species) is a highly toxic plant that contains aconitine and related alkaloids. Aconite roots have been used in traditional Chinese medicine, only after processing, which reduces the toxic alkaloid content by up to $90 \%$, for its analgesic, anti-inflammatory, and cardiotonic effects. Soaking and boiling during processing or decoction preparation will hydrolyze aconite alkaloids into less toxic and non-toxic derivatives. Inadequate processing, consumption of large amount, or ingestion of the raw plant will increase the risk of toxicity. ${ }^{4}$ Aconite poisoning is more common in East Asia. Recognition of this entity may pose a considerable challenge in both diagnosis and management, particularly in parts of the world where aconite use is less common. ${ }^{4}$ There are many different species of aconitum. In Europe and North America, aconite poisoning usually occurs after ingestion of aconitum napellus, commonly called monkshood, wolfsbane, or devil's belmet. ${ }^{4}$

Ingestion of any part of the aconite plant or its extracts can cause cardiotoxicity, neurotoxicity, and gastrointestinal (GI) manifestations. ${ }^{4}$ Aconite binds to the open state of voltage-sensitive sodium channels and induces its persistent activation in the myocardium and the nervous system, rendering cells refractory to further excitation. Tachydysrhythmias occur predominantly because of triggered activity due to delayed and early afterdepolarization within the cardiac myocytes. ${ }^{4}$

Bidirectional ventricular tachycardia from aconite poisoning has been previously reported in North America. ${ }^{3}$ The clinical course has been characterized by refractory ventricular dysrhythmias often resolving after 24 hours, but, in some cases, complete recovery may be delayed beyond a week post-ingestion. ${ }^{5}$ The mainstay of treatment is supportive care and management of ventricular dysrhythmias with standard Advanced Cardiac Life Support protocols. There is no antidote for aconite toxicity, but there have been anecdotal reports of successful restoration of sinus rhythm with anti-dysrhythmics, specifically those with sodium channel blocking properties: flecainide, lidocaine, procainamide, and amiodarone. ${ }^{5}$ If ventricular dysrhythmias and hemodynamic compromise are refractory to medical management, early use of extracorporeal membrane oxygenation is recommended., ${ }^{4,5}$ The aconitine alkaloids are rapidly absorbed from the GI tract and therefore GI decontamination, gastric lavage, or activated charcoal may only be considered in patients who present early to the ED with careful consideration of aspiration risk and airway protection. Enhanced elimination via extracorporeal techniques (e.g., hemodialysis) is unlikely to be effective in removing circulating aconitine due to its lipid solubility and large molecular size. ${ }^{4,5}$

\section{CONCLUSION}

This case highlights the ECG findings of bidirectional ventricular tachycardia. Emergency physicians should be aware that the differential diagnosis of this unmistakable rhythm is digoxin toxicity, catecholaminergic polymorphic ventricular tachycardia, and herbal aconite poisoning. Digoxin toxicity is at the top of the differential diagnosis of bidirectional ventricular tachycardia and should be empirically treated while further assessment and diagnostic testing are considered.

\section{Competing interests}

None declared.

\section{$\underline{\text { Meetings }}$}

This work was presented as an abstract poster presentation in the North American Congress of Clinical Toxicology (NACCT) in Chicago, IL, October 27, 2018.

\section{$\underline{\text { Disclosure }}$}

For the purpose of publishing this case report, we were unable to reach family for consent. 


\section{REFERENCES}

1. Richter S, Brugada P. Bidirectional ventricular tachycardia. 7 Am Coll Cardiol 2009;54(13):1189.

2. Femenia F, Barbosa-Barros R, Sampaio SV, et al. Bidirectional ventricular tachycardia: a hallmark of catecholaminergic polymorphic ventricular tachycardia. Indian Pacing Electrophysiol 7 2012;12(2):65-8.
3. Smith SW, Shah RR, Hunt JL, Herzog CA. Bidirectional ventricular tachycardia resulting from herbal aconite poisoning. Ann Emerg Med 2005;45(1):100-1.

4. Chan TYK. Aconite poisoning. Clin Toxicol (Pbila) 2009;47 (4):279-85.

5. Coulson JM, Caparrotta TM, Thompson JP. The management of ventricular dysrhythmia in aconite poisoning. Clin Toxicol (Phila) 2017;55(5):313-21. 